\title{
Oral Health and Rehabilitation among the
}

\section{Institutionalized Elderly}

\author{
Nélio Veiga ${ }^{\mathrm{a}} *$, Liliany Diniz ${ }^{\mathrm{b}}$, Carlos Pereira ${ }^{\mathrm{c}}$, Odete Amaral $^{\mathrm{d}}$, Inês Coelho ${ }^{\mathrm{e}}$, \\ Joana Pereira ${ }^{\mathrm{f}}$ \\ ${ }^{a}$ Health Sciences Institute - Universidade Católica Portuguesa. Estrada da Circunvalação, 3504-505, Viseu, Portugal. \\ nelioveiga@gmail.com \\ ${ }^{b}$ Health Sciences Institute - Universidade Católica Portuguesa. Estrada da Circunvalação, 3504-505, Viseu, Portugal. \\ lily_dinis@hotmail.com \\ ${ }^{c}$ CI\&DETS - Polytechnic Institute of Viseu. Campus Politecnico, 3504-510,Viseu, Portugal. carlospereiraviseu@gmail.com \\ ${ }^{d}$ CI\&DETS - Polytechnic Institute of Viseu. Campus Politecnico, 3504-510,Viseu, Portugal. mopamaral@gmail.com \\ ${ }^{e}$ Family Health Unit Grao Vasco. Avenida Madre Rita Amada Jesus, 3500-179, Viseu, Portugal. inesisabelcoelho@gmail.com \\ ${ }^{f}$ Riga Stradins University. Street Dzirciema, LV-1007, Riga, Latvia. jitpereira@hotmail.com

\begin{abstract}
The aging and the elderly are highly associated with limitation or dependency, with some loss of capacities and the gain of conditions or age-associated pathologies. Despite advances in preventive dentistry, edentulism and oral diseases are major public health issues in Portugal, mainly among the elderly. This study investigated the oral status and level of oral rehabilitation among Portuguese institutionalized elderly. The purpose of the study was to determine the prevalence of edentulism and assess the oral rehabilitation and oral/prosthetic hygiene habits in a sample of institutionalized elderly using a cross-sectional study in a sample of 118 institutionalized elderly. Data collection was performed by applying a questionnaire about oral health behaviors and through an intraoral observation in order to determine the oral status and verify the condition of the oral rehabilitation of each participant. The findings revealed that $78.6 \%$ have a number of decayed, missing and filled teeth more than $20,58.8 \%$ presented total edentulism (with no natural teeth in the oral
\end{abstract}

* Corresponding author. Tel.: +0-000-000-0000 ; fax: +0-000-000-0000

E-mail address: nelioveiga@gmail.com 
cavity) and $66.7 \%$ had a removable prosthesis. Only $44.1 \%$ practiced daily oral/prosthetic hygiene at least twice a day while $29.0 \%$ and $67.7 \%$ complained of toothache and difficulty in chewing respectively. The educational level of the elderly was associated with dental pain $(\mathrm{p}=0.012)$ and oral/prosthetic hygiene $(\mathrm{p}=0.034)$. A high prevalence of tooth loss was found in the sample studied and also the need to improve the removable prosthesis is fundamental. Improvement in oral health care and oral hygiene habits is essential to promote better oral health and quality of life among the institutionalized elderly.

C 2016 Published by Future Academy www.FutureAcademy.org.uk

Keywords: Separated by semicolons ; Times New Roman 8

\section{Introduction}

In the last century, the world has witnessed an unparalleled decline in mortality rates, causing a considerable increase among the human population. Increased life expectancy is one of the greatest achievements of mankind. The medical and scientific communities have as their primary objective the continuous maintenance of the quality of life of the senior citizens (Lunenfeld \& Stratton, 2013; Veras, 2009; Lutz et al., 2008). Thus, there is an increased incidence of disorders that last for years, with a need for care, treatment and constant medication. This situation is directly associated with aging and therefore, a challenge for both the senior citizen and the family, reflecting an increased burden on health systems and specialized units in the comprehensive care of these patients (Veras, 2009).

The World Health Organization (WHO) points to the senior population as an important target for oral health care. The causes of aging, directly or indirectly, increase of the risk of oral disease development and the presence of problems in the overall health associated with chronic diseases or drug therapy that can be the source of various oral manifestations (Dolan et al., 2005; Ghezzi \& Ship, 2000).

There are several prevalent oral conditions in geriatric patients: dentition status changes, root caries, periodontal support loss and tooth loss affecting the functional and aesthetic levels. Conditions related to the use of prosthesis, oral lesions, oral malignancy, xerostomia, pain and craniofacial discomfort are also considered frequent complications in the elderly that decrease the quality of life of patients and should be efficiently diagnosed and treated (Shay, 2002; Ghezzi \& Ship, 2000).

The general deterioration of health as well as the physical and psychological state of the elderly 2354 
brings us to the need to maintain their quality of life. The preservation of the masticatory capacity and taste, verbal expression and aesthetics are important factors for the personal and social performance of these patients and should be valued and achieved through regular oral health care (Shay, 2002).

However, there are several obstacles that stand between the institutionalized elderly and access to oral health care, namely, the high cost of the treatments, the lack of perception of their needs, the lack of education for oral health and the lack of preparation of health professionals (Dolan et al., 2005; Fiske et al., 2000).

Within the senior population, individuals from disadvantaged or institutionalized socioeconomic strata may present an increased risk of oral disease development by the fact that there is an association with the presence of a weak general health or some degree of functional limitation (Vargas et al., 2001).

The challenge is to overcome the remaining obstacles between the geriatric patient and oral health care and create appropriate strategies and policies that can educate professionals and geriatric doctors and caregivers to improve the oral health of this population (Dolan et al., 2005).

The WHO has presented a program to improve oral health in the twenty-first century, which states that oral health must not be confined to the existence of healthy teeth, but also involve the absence of orofacial chronic pain, oral or pharyngeal cancer, lesions in the oral tissues, oral birth defects or other problems or complications that affect the dental, oral and craniofacial tissues (Petersen, 2003).

Although oral health should be seen as a whole which surrounds the craniofacial complex, it should not be undervalued as it contributes to the two most prevalent oral diseases in the world, and very common among the elderly - dental caries and periodontal disease. Edentulism, in turn, is still very present in adults over 65 years in many industrialized countries (Petersen, 2003). 


\section{Problem statement}

Despite advances in preventive dentistry, edentulism and oral diseases remain major public health issues in Portugal, mainly among the elderly. This age group is characterized, in general, by a lack of oral health care thanks to the lack of knowledge, needing perception or by the presence of obstacles (financial, physical, among others) that create difficulties among the senior citizens' access to an oral health specialist.

\section{Research questions}

What is the oral status and level of oral rehabilitation among Portuguese institutionalized elderly?

\section{Purpose of the study}

This study aimed to determine the prevalence of edentulism and assess the oral rehabilitation and oral/prosthetic hygiene habits in a sample of institutionalized elderly.

\section{Research methods}

An epidemiological observational cross-sectional study was designed to identify the most prevalent oral conditions among the institutionalized elderly.

This study is based on a sample of institutionalized elderly from three different cities of Portugal (Viseu, Arganil and Porto). Inclusion criteria were established: be an institutionalized elderly, age equal or higher than 65 years who were willing to participate in the study. The sample integrated all residents who fulfilled these requirements, regardless of the level of autonomy, so that the reliability of the study would be as close as possible to the existing reality in any residential institution for elderly.

We obtained a final sample of 118 institutionalized elderly (Viseu=68, Arganil=25, Porto=25) in which the average age was $78.3 \pm 12.0$ years old.

For data collection, a questionnaire was used containing items about socio-demographic variables, general health, oral health and nutritional habits. The data collection was complemented by an intraoral observation for oral health assessment and level of oral rehabilitation of the elderly, 2356 
using disposable material such as gloves, masks and intraoral observational mirrors. The decayed, missing and filled permanent teeth index (DMFT) was defined for each participant in the study to assess oral health.

The statistical analysis was performed with the SPSS software version 22.0® (IBM Corporation, New York, USA). Absolute and relative frequencies were applied in the analysis of quantitative variables. To analyze the associations between variables, the independence Chi-square and Fisher's exact tests were applied, where the level of significance was 0.05 .

\section{Findings}

The final sample consisted of 118 individuals aged between 50 and 96 years living in Viseu, Porto and Arganil, in which $76.3 \%$ were women while $23.7 \%$ were men. Analyzing the educational level of the sample, we can verify that the majority did not have a high educational level: $18.9 \%$ could not read, $35.7 \%$ had completed the fourth grade and $32.1 \%$ had not even completed the fourth grade.

Of the total sample, $58.8 \%$ presented total edentulism with no natural teeth and, among the elderly with total edentulism, $66.7 \%$ had a removable prosthesis. Only $44.1 \%$ practised daily oral/prosthetic hygiene at least twice a day. $29.0 \%$ refer complained of toothache; $58.1 \%$ complained of dry mouth and $67.7 \%$ had difficulty in chewing. $78.6 \%$ of the elderly presented a total score of decayed, missing and filled teeth (DMFT) higher than 20.

All participants said that they never using dental floss, and $90.2 \%$ admitted not having a dental appointment in the last 12 months, leaving only 4 participants having had a dental appointment due to prosthetic problems. By analyzing the dental prosthesis, it was found that the proportion of lower and upper dentures is very similar ( $82.5 \%$ and $84.5 \%$, respectively).

In the case of the effect of the educational level in DMFT, there were no statistically significant differences $(p=0.505)$. However, the educational level of the elderly was associated with dental pain $(\mathrm{p}=0.012)$ and oral/prosthetic hygiene $(\mathrm{p}=0.034)$. Within the study sample, the percentage of individuals using prosthesis $(54.6 \%)$ was very close to the percentage of non-users $(45.4 \%)$, but 
when analyzing the differences between the use of prosthesis and educational level, no statistically significant differences were found $(\mathrm{p}=0.504)$.

\section{Conclusions and Implications}

A high prevalence of tooth loss was verified among the sample studied and also the need to improve the removable prosthesis was found to be critical. The main causes of tooth loss are related with the development of severe dental caries (which among the elderly consist mostly of root caries) and advanced periodontal disease causing loss of tooth support. The absence of a large number of teeth cause serious chewing difficulties which limits food choice and may cause nutritional problems. Missing teeth implies a decrease in the level of self-esteem, lack of aesthetic and discomfort causing social mismatch and depressive states (Northridge et al., 2012).

Improvement in oral health care and oral hygiene habits is essential to promote better oral health and quality of life among the institutionalized elderly. The society of today is characterized by an aging population, especially in developed countries. This pattern leads to the need to determine better health care specifications by adapting them to the real needs of the senior patient. The big challenge will be to find a way to deal with chronic diseases that affect this subpopulation as well as their consequences (Kandelman et al., 2008)

Although patients have prosthetic rehabilitation, in many cases these devices are, in general, maladaptive, not fulfilling their functionality and causing pathological changes in the soft tissues, namely, denture stomatitis. There is a lack of perception for the need for treatment and oral care which can be verified in the elderly patients who do not seek dental health professionals to readjust or make a new prosthesis when required (Preshaw et al., 2011).

With regard to oral health, there are still some problems closely associated with advanced age, derived from the etiologic agents of the edentulous, such as, dental caries, periodontal disease, xerostomia and pathologies associated with soft tissue. Poor oral health can affect the quality of life of the elderly, being a public health issue that requires the creation of strategies and specific oral health programs appropriate to this age group. Studies like this one have a significant relevance to expose the main problems by providing a theoretical and scientific support based on real cases that will allow the implementation of policies and strategies to decrease gaps in the maintenance of oral health among the elderly. 
These oral health programs for the elderly patient should prioritize the implementation of preventive measures against the major risk factors and measures of oral health promotion and education. These programs should not only target the elderly but also the health professionals and caregivers responsible for these seniors, in order to make them aware of the importance of oral health in general health (Fiske et al., 2000).

A high proportion of the elderly present a very low access to dental appointments, lack of information about the importance of oral health as an integral part of general health, physical inability to travel to a dental office and financial incapacity to acquire oral health treatments needed. Furthermore, and for the institutionalized elderly, a dental monitoring program including diagnosis and evaluation, with communication with medical specialists and caregivers who accompany each patient to create a detailed and complete medical history, specifying the conditions, medication, habits and medical and family history is essential. The visit to the dental office should be provided and facilitated by the institution as in the medical care of other health fields. A complete treatment plan, aimed at the best options for each individual providing the restoration of oral health and quality of life should be enforced. Oral health must be made affordable and accessible to all seniors, regardless of their degree of limitation or dependency and the severity of their needs.

\section{Acknowledgments}

The authors are deeply indebted to the researchers that participated in the development of this study and data collection phase: Prof. Paulo Melo, Dr. Ana Herdade and Dr. Carina Coelho. We also thank the institutionalized elderly, directors and workers of the nursing homes for their important contribution for this study.

\section{References}

Dolan, T.A., Atchison K., \& Huynh T. (2005). Access to dental care among older adults in the United States. $J$ Dent Educ, 69(9), 961-974.

Fiske, J., Griffiths, J., Jamieson, R., \& Manger, D. (2000). Guidelines for oral health care for long-stay patients and residents. Gerodontology, 17(1), 55-64.

Ghezzi, E.M., \& Ship, J.A. (2000). Systemic diseases and their treatments in the elderly: impact on oral health. J Public Health Dent, 60(4), 289-296.

Kandelman D., Petersen PE., \& Ueda H. (2008). Oral health, general health, and quality of life in older people. Spec Care Dent, 28(6), 224-236.

Lunenfeld B. \& Stratton P. (2013). The clinical consequences of an aging world and preventive strategies. Best 
Pr Res Clin Obs Gynaecol, 27(5), 643-659.

Lutz W., Sanderson W., \& Scherbov S. (2008). The coming acceleration of global population ageing. Nature, 451(7179), 716-719.

Northridge ME., Ue FV., Borrell LN., De La Cruz LD., Chakraborty B., Bodnar S., Marshall S., \& Lamster IB. (2012). Tooth loss and dental caries in community-dwelling older adults in northern Manhattan. Gerodontology, 29(2), 464-473.

Petersen PE. (2003). The World Oral Health Report 2003: continuous improvement of oral health in the 21st century-the approach of the WHO Global Oral Health Programme. Community Dent Oral Epidemiol, 31(Suppl 1), 3-24.

Preshaw P., Walls AW., Jakubovics N., Moynihan P., \& Jepson NJ. (2011). Association of removable partial denture use with oral and systemic health. J Dent, 39(11), 711-719.

Shay K. (2002). Infectious complications of dental and periodontal diseases in the elderly population. Clin Infect Dis, 34(9), 1215-1223.

Vargas C., Kramarow E., \& Yellowitz J. (2001). The oral health of older Americans. Aging Trends, 188(3), 18

Veras R. (2009). Envelhecimento populacional contemporâneo: demandas, desafios e inovações. Rev Saude Publica, 43(3), 548-554. 